\title{
INTERNATIONAL ASSEMBLY
}

\section{DISCUSSES MECHANISMS}

\section{OF DNA DAMAGE, REPAIR}

For five days, 60 invited speakers, 115 contributors and more than 200 additional attendees probed the mysteries of DNA at the International Conference on "Mechanisms of DNA Damage and Repair: Implications for Carcinogenesis and Risk Assessment," held at NBS.

Donald R. Johnson, director of NBS' National Measurement Laboratory and the chairman of the organizing committee, ${ }^{1}$ opened the June 2-7 conference and introduced Ernest Ambler, director of NBS, who greeted the distinguished gathering of foremost experts in the field. The conference chairman, Michael G. Simic, raised the question: "How do we protect ourselves from natural and man-created hazards which damage DNA, and to which we are exposed on a daily basis?"

Meeting for the first time to exchange ideas and perceptions of future trends were scientists dealing with topics as diverse as DNA and protein damage, DNA repair enzymes, the consequences of unrepaired DNA or faulty repair of DNA, site-specific DNA binding drugs, dosimetry of exposure to genotoxic agents, DNA damage in risk assessment, and the role of science in regulatory decisions.

The conference was subdivided into an introduction, three scientific sections, and a poster exhibition. The three sections "Mechanisms of DNA Damage," "DNA Repair and Consequences," and "Risk Assessment" were chaired by distinguished veterans in those fields: George Scholes, Larry Grossman, and Arthur D. Upton, respectively. Each section was further subdivided into half-day sessions. In all of the sessions the primary objective was to discuss the mechanisms of phenomena at the molecular level.

As was pointed out repeatedly, we are exposed to many actual and potential hazards. It is now becoming increasingly clear that we cannot devote all our resources to the investigation of hazardous aspects of all agents to which we are or might be exposed. Consequently, mechanistic understanding of deleterious processes is becoming necessary to assure predictability

\footnotetext{
${ }^{1}$ Also made up of C. Reimann, S. A. Miller, D. Longfellow, E. Clementi, M. Kasha, P. Cerutti, G. Scholes, J. M. Brown, and E. L. Powers.
}

with high degree of certainty. Hazardous agents can have numerous consequences. The conference dealt predominantly with one of these consequences-cancer, and the role of DNA in carcinogenesis.

The mechanisms of carcinogenesis are not clear at present, in spite of tremendous advances made in the last decade. However, DNA is recognized as one of the major participants in at least one step of what has been recognized as a multistep process. This multiplicity of steps makes the study of the mechanisms of carcinogenesis extremely complex. It also renders prediction of the carcinogenicity of agents unreliable, particularly for weak carcinogens. From the point of view of safety, should we pay more attention to initiators or to promoters?

Conclusions based on available statistics and accidental human exposure are inadequate because comprehensive information is rarely available and accidents are statistically small in size. This has prompted extensive use of animal studies. Unfortunately, direct extrapolation from animal models to humans is, in many cases, difficult. For example, in a well publicized case, BHT has been demonstrated to be carcinogenic in rats only.

The other major issue in carcinogenesis is the acceptable level of exposure. Can one extrapolate the carcinogenic effect down to low levels of exposure, or is there a "threshold" below which the exposure does not cause cancer?

Similar questions were raised by Dr. Ambler in his opening remarks. He pointed out the widespread use of ionizing radiations in medicine and technology. He reminded the audience of the prospective exposure to radiations in space of personnel associated with gravitationless technologies. He also raised concern about the danger of radon as a potential carcinogen. The carcinogenic nature of radon is indicated by statistical studies, and the presence of this insidious gas in our homes is of considerable concern. He pointed out the long tradition of NBS in the measurements of ionizing radiations, and the necessity to develop new measuring approaches, through mechanistic studies of DNA damage and repair, for an accurate assessment of the exposure levels of humans to ionizing radiations. These studies would assist us not only in critically evaluating the extrapolation 
of the effects of low radiation dose levels, but also in manipulating those effects in our favor by preventive measures and treatments.

In his keynote address, Rep. Don Ritter of Pennsylvania talked about the hazards of an advanced technological society. He indicated that risk assessment is one of the critical aspects of hazards control that our society cannot ignore. He appealed to the scientists to work towards a sound scientific basis for risk assessment. Risk must be quantified by accurate measurements (e.g., risk assessment dosimetry). For fiscal reasons we shall never be able to protect ourselves from all potential hazards. Choices based on reliable scientific predictions must be made so that we can protect ourselves preferentially from the most hazardous exposures, he said.

...we shall never be able to protect ourselves from all potential hazards. Choices based on reliable scientific predictions must be made...

The importance of science in risk assessment and the associated measurements of exposure to hazardous materials was also emphasized in the introductory presentation of David P. Rall, director of the National Institute of Environmental Health Sciences at Research Triangle, NC. He also mentioned the extensive activities in these areas that are being conducted at his institution.

The final opening remarks were given by Richard H. Adamson, director of Cancer Etiology at the National Cancer Institute in Bethesda, MD. He emphasized the very latest development in carcinogenesisoncogene research. Understanding the mechanisms of oncogene activation may become the key to prevention and treatment of cancer.

Session I dealt with the mechanisms of free radical reactions in model systems. Free radicals have been implicated in chemical carcinogenesis. However, related free radical reactions have not been identified. Free radicals generated by ionizing radiations are responsible for DNA damage and its consequences. The significance of different radicals in inactivation of $\phi \times 174$ DNA was discussed by J. Blok. New kinetic and mechanistic information regarding DNA base damage, strand breaks, and crosslinking was presented (M. G. Simic). The yield of DNA strand breaks was shown to be dependent on fast chemical repair by intracellular thiols (B. D. Michael). Direct ionization of bases by lasers can also result in strand breaks (D. Schulte-Frohlinde). The photoionization approach allows kinetic and mechanistic treatment of damage induced by the direct action of radiation. Photoionization and chemically induced ionization (oxidation) (Jovanovic and Simic) are novel approaches in the study of direct effects, yet are of primary importance in understanding the biological effects of ionizing radiations.

Direct free radical detection by ESR was presented by $M$. Symons and discussed by J. Herak. Analysis of radiolytic products was elaborated by J. Cadet. Inactivation of enzymes by free radicals was touched upon by $R$. $L$. Willson. It may be concluded from this session that time-resolved techniques, such as pulse-radiolysis and laser photolysis, coupled with product analysis, will play an important role in the study of free radical processes in biology and medicine in the next decade.

Sessions III and IV (the Session II discussion is reported later in this account) dealt with mechanisms of DNA damage in cells by various drugs, chemicals, ionizing radiation, and autoxidation processes. Crosslinking within DNA, between DNA and proteins, and various approaches to measure crosslinks showed signs of renewed interest (K. Kohn). Renewed interest in lightand radiation-induced crosslinks was evident from the special poster session (A. Cress, L. Mee, and S. J. Adelstein; M. G. Simic and M. Dizdaroglu; M. J. Peak and J. G. Peak) which was reviewed by N. L. Oleinick. However, the biological consequences of crosslinking are not yet fully understood, except for the crosslinks generated by light-induced cyclobutane dimer for-

Other agents, often described as tumor promoters, cause a similar (prooxidant) state, and it may be that the prooxidant state itself serves to promote tumorigenesis...

mation (thymine dimer, psoralin, etc., in Session V). D. B. Ludlum presented mechanisms of DNA modification and repair induced by mutagenic sulfur mustards and nitrosourea. The sequence specificity of anthramycins and other compounds that bind covalently in the minor groove was explored by L. H. Hurley, using defined DNA sequences, the techniques of "footprinting," and Maxam-Gilbert sequencing. Carcinogens of various kinds also show selectivity in their action, reflecting the base composition, nucleotide sequence or conformation of DNA, the structure of chromatin, or transcriptional activity of different genes (G. N. Wogan).

One effect of radiation is to produce a prooxidant state in cells. Other agents, often described as tumor promoters, cause a similar state, and it may be that the prooxidant state itself serves to promote tumorigenesis, after initiation has ocurred (P. A. Cerutti). The mech- 
anisms may require modulation of regulatory genes, mediated perhaps by poly ADP ribosylation which is itself sensitive to the prooxidant state.

In Session IV, (mainly about the effects of ionizing radiations), J. F. Ward indicated the nonhomogeneity of damage and discussed some of its consequences. For example, he demonstrated that it is impossible to distinguish true double strand breaks which involve base destruction on both strands and loss of genetic information from single strand breaks on the two strands offset by a few bases (which are, in principle, repairable without loss of information). On a related topic, P. E. Bryant showed that some double strand breaks arise from the action of repair enzymes on base damage opposite a single strand break. He described a model system for double strand breaks in which these are created intracellularly by the action of restriction endonucleases. $\mathrm{J}$. Lett has studied the eventual appearance of DNA damage in nondividing rabbit retina cells years after irradiation and apparent recovery of the DNA from the immediate effects of radiation. R. E. Meyn described differences in the yield of ionizing radiation damage in different tissues in vivo (perhaps reflecting relative levels of hypoxia), and also varying capacity to repair damage.

Strand breaks created by enzymic incision are present only transiently after UV damage (K. Erixon). DNA synthesis inhibitors cause incomplete repair sites

...double strand breaks can be induced by $\mathrm{Fe}$ and $\mathrm{Cu}$ ions, hydrogen peroxide, and physiological reductants, mimicking the multiple damage sites induced by radiation.

to accumulate, leading to high levels of breaks. M. Chevion showed that double strand breaks can be induced by $\mathrm{Fe}$ and $\mathrm{Cu}$ ions, hydrogen peroxide, and physiological reductants, mimicking the multiple damage sites induced by radiation.

Section 2, subdivided into Sessions V-VII, dealt with the mechanisms of enzymatic repair of damaged DNA. (Chemical repair of damaged DNA at the free radical stage was not part of the conference program since it was already discussed at the conference on "Radioprotectors and Anticarcinogens," held at and sponsored by NBS in 1982.)

In the first session on the repair of DNA damage, D. Mount reviewed the regulation of excision repair and SOS repair in $E$. coli. He described a lex. $A$ mutant that produces an unstable repressor of repair genes, leading to increased expression of $u v r A, B$, and $D$. Excision is consequently elevated in these cells. The $u v r A, B, C$ genes have been cloned (L. Grossman), and used to produce large quantities of protein products. Excision repair activity was tested in vitro, utilizing a pyrimidine dimer in a specified sequence. Cuts are made on each side of the dimer, but the oligonucleotide is released only when helicase II ( $u v r D$ gene product) and DNA polymerase I are present with dNTPs.

The repair of dimers by $M$. luteus $\mathrm{UV}$ endonuclease entails a two-step incision (R. H. Grafstrom). The Nglycosylic bond of the $5^{\prime}$ pyrimidine of the dimer is broken, leaving the dimer attached to the backbone via the $3^{\prime}$ base; the resulting apyrimidinic site is then cleared by an endonuclease activity. $M$. luteus and T4 endonucleases are similar. The gene coding for the latter, den $V$, has been cloned and introduced into $E$. coli, yeast, and the UV sensitive CHO mutant UV-5 (J. K. de Riel). In all organisms studied, excision repair was partially or completely restored.

E. C. Friedberg described the $R A D$ genes of yeast. Five have been cloned, and all found necessary for the incision of DNA after UV induced damage. Four have been completely sequenced, and mutation at specific sites has defined regions essential for repair. Two genes have been mapped on particular chromosomes. $R A D$ genes do not complement $u v r A, B, C, D$ mutations in $E$. coli.

Two repair activities were featured in T. Lindahl's talk-a glycosylase, which acts on various altered forms of thymine, and the methyl-transferase or Ada protein from $E$. coli. This protein has two acceptor sites for methyl groups, with one accepting from $0^{6}$-methylguanine, and the other from phosphotriesters. It is possible that the second reaction is involved in autoregulation, activating the ada gene and inducing an adaptive response.

Some repair enzymes that appear to be effective against low doses of damaging agents can be overwhelmed at high doses because the enzymes cannot be replaced by cells fast enough. Therefore, if the enzymatic system functions properly, cancer will not result from low, chronic doses of certain kinds of carcinogens.

Metabolic alterations of DNA were discussed by B. Weiss. The presence of thymine, rather than uracil, in DNA may reflect the common occurrence of spontaneous deamination of cytosine in DNA, creating uracil. If uracil were a standard constituent of DNA, a repair mechanism would have to distinguish "accidental" from "intended" uracils. Mutants of $E$. coli lacking dUTPase are unable to make dTTP, and have been used to examine the effects of replacing thymine with uracil in the DNA. 
The $u m u D C$ operon in E. coli is involved in $\mathrm{mu}-$ tagenesis (L. Marsh). It, and its plasmid analogue, mucAB, have been cloned and sequenced. A model for its action proposes that during replication, polymerase III becomes blocked at the site of damage, but that it can interact with $u m u D C$ and recA products. This allows polymerization to continue with the risk of introducing alterations at the site of damage.

Apurinic (AP) sites are common intermediates in mutagenesis (L. A. Loeb). In a phage reversion assay, it was found that the predominant DNA sequence alteration was the substitution of deoxyadenosine opposite the AP site. Possible AP sites are common intermediates in mutagenesis: replication may stop at a bulky adduct until the adduct is removed, leaving an AP site, at which point the polymerase resumes synthesis, and an adenine is introduced.

Mistakes in normal replication-mismatches-are likely to lead to mutation unless the repair process can discriminate newly synthesized from parental strands. Discrimination might be on the basis of methylation (new strands being unmethylated), and P. Modrich confirmed that in $E$. coli mismatch correction occurs on the unmethylated strand. In his model, a methyl group acts as a reference point for the repair system. Mismatches do not occur only through faulty replication. Common mistakes might result, for example, from the conversion of methylated cytosine to thymine rather than cytosine, giving a GT base pair; these are efficiently recognized

In higher organisms, recombination repair of double strand breaks may be the origin of sister chromatid exchange.

and restored to the correct form in an E. coli assay system using defined heteroduplex DNA molecules (M. Radman). The cell may be programmed to recognize such mispairings and to make the correction on the right strand. Mismatch repair occurring on both strands may account for double strand breaks. In higher organisms, recombination repair of double strand breaks may be the origin of sister chromatid exchange.

F. Hutchinson reviewed the classification of mutations. Targeted mutations depend on a lesion in the particular gene to initiate the mutational event; nontargeted mutations can occur in undamaged DNA, and result from an infidelity of replication. Different agents produce different spectra of mutations-transitions (e.g., GT), transversions (e.g., AG), frameshifts, large deletions/insertions, and double events. Spontaneous mutations in the lac I gene of $E$. coli have been examined for evidence of sequence specificity (B. W. Glickman). There is a frameshift hotspot in the region of tandemly repeated tetramer. Deletions tend to occur where repeated sequences lend stability to a misalignment. Single base frameshifts may result from DNA repair.

Bridging the gap between prokaryotic and eukaryotic organisms, $\mathbf{M}$. Seidman described the use of shuttle vector plasmids, able to replicate in both bacteria and mammalian cells. Plasmids treated with DNAdamaging agent are introduced into mammalian cells, where replication, repair, and mutation occur. A marker gene on the plasmids allows monitoring of these processes by assaying the appropriate activity (coded by the marker gene) in a bacterial host. Mutations introduced in the plasmid can be characterized in terms of sequence changes. The novel plasmid described by Seidman has a low rate of spontaneous mutation. $\mathrm{K} . \mathrm{H}$. Kraemer used an expression vector plasmid, containing the gene for chloramphenicol acetyltransferase. Pretreatment of plasmid with UV before introducing it into human cells caused a decrease in CAT activity, with the extent of the decrease reflecting the DNA repair phenotype of the cells. Thus, UV inactivation of CAT expression was greatest in XP cells.

L. D. Samson described an adaptive response to alkylating agents in certain mammalian cell lines. Pretreatment with a low level of alkylating agent renders cells resistant to a subsequent acute, high dose. She ascribed this important effect to an increase in $0^{6}$-methylguanine transferase activity. An antibody has been produced to the $E$. coli methyltransferase, and this has been used to identify putative human methyltransferase.

A general response to severe DNA damage is the stimulation of poly(ADP-ribose) synthesis, with consequent reduction in NAD and ATP concentrations in the cell. N. A. Berger suggested that the disruption of energy-dependent processes such as RNA, DNA, and protein synthesis might serve as a suicide response, killing the cells to protect the organism from possible mutational effects.

H. Zarbl and M. Barbacid described investigations into the role of ras oncogenes in carcinogenesis-in particular, the question of whether oncogene activation is an effect, rather than a cause, of carcinogenesis. In tumors induced by a single treatment with a chemical carcinogen, transforming $\mathrm{H}$-ras oncogene is very common, and the activation (i.e., base alteration) is at a particular point in the DNA sequence. The type of change is specifically related to the carcinogen used, which suggests that the activation is a direct result of carcinogen damage rather than a secondary effect of carcinogeninduced transformation. Another oncogen c-myc, be- 
comes activated not by a point mutaition, but rather by translacation, proviral integration, or amplification, giving high levels of c-myc expression (M. D. Cole). Interestingly, interferon can cause reversion of the transformed phenotype, and the oncogene is then no longer expressed. When the transformed cell was mer- (i.e., unable to repair $0^{\circ}$-methylguanine), the nontransformed revertants produced with interferon treatment had restored methyltransferase activity and were resistant to alkylating agents (R. S. Day). Thus, there appears to be a close link between oncogene activation and the merphenotype. M. Barbacid indicated that $\mathrm{G} \rightarrow \mathrm{A}$ change by NMU can activate an oncogene in a much earlier stage of multistep cancer development than previously believed.

An important assessment of the current activities in the study of mutagenic changes on DNA, and a novel approach to these studies, was suggested by $\mathbf{M}$. Radman. Complete sequencing of DNA is an enormous task that cannot be achieved by present techniques. Rather than investing enormous resources into it, he suggested development of methodologies which would decode the sequence differences only.

The question of what to do about faulty repair and the sequences which may lead to cancer was addressed in Session II dealing with DNA binding agents. In his introductory remarks, J. W. Lown indicated the potential importance of site specific DNA binding drugs in cancer treatment. J. B. LePecg described novel molecules whose remarkably high affinity for DNA derives

Computer modeling allows the study of possible inter-

actions between side-chatins of intercalators and particular

DNA sequences in designing new anti-tumor drags.

from a dimeric or trimeric arrangement of intercalating moieties. Neocarzinostatin binds to DNA by intercalation and causes DNA damage-strand breaks, release of free base, and covalent adducts of both alkalilabile and highly stable forms (I. H. Groldberg). Computer modeling allows the study of possible interactions between side-chains of intercalators and particular DNA sequences in designing new anti-tumor drugs (S. Neidle). The anti-tumor drug netropsin does not intercalate with DNA, but rather binds in the minor groove of the helix. The specificity and geometry of this binding have been the subject of studies by $x$-ray crystallography (R. E. Dickerson).

If specific sequences could be identified in oncogenes or in the DNA of cancer cells, then site-specific drugs could be designed either to prevent de velopment of cancer or to kill cancerous cells. This elegant approach could become an important solution in aur continuing fight against carcinogenesis.

Section 3 on Risk Assessment was introdoced by Sanford A. Miller, director of the center for Safety and Applied Nutrition of the FDA and a member of the conference organizing committee. He pointed out the difficulties associated with safety assessment of foods and the highly responsible role of FDA in ensuring the safety of our food supply. One should not forget, of course, that classical processing of foods or even natural foods can be detrimental (see, e.g., Dietary Carcinogens and Anticarcinogens, by B. Ames, Science, 221,1256, 19B3).

Session WIII dealt with the measurament of exposure to genotoxic compounds. Classical aronatic adducts to DNA were discussed by F. Kadlubar, and W. G. Thilly talked about how to discriminate different patterns of genetic change induced by different mutagens. Steve R. Tannenbaum, the chairman of the session, presented the use of blood proteins as alkylation dosimeters in general exposure dosimetry. H. Bartsch talked about population studies in areas of Japar, China, and India having little population migration, and in which urine tests were used to determine levels of endogenous formation of $\mathrm{N}$-nitroso compounds and to learn how these levels are affected by dietary components and modifying chemicals.

J. D. Groopman presented the use of monoclonal antibodies for detection of carcinogens and their adducts, which are produced by molds in foods, fuel combustion, and cigarette smoking. S. S. Wallace and R. A. Floyd told of novel approaches in the measurement of thymine glycol and 8-hydroxyguanine, which are two major products of DNA base damage.

The Scientific Basis for Genotoxic Risk Assessment, Session IX, was chaired by P. N. Magee. P. C. Hanawalt described repair of DNA as a selective process. Cells of xeroderma pigmentosum show a low level of UV excision repair, but this response is heterogeneous. Certain DNA domains are repaired at normal rates, while the rest of the DNA shows deficient repair. The repetitive $\alpha$ DNA in African Green Monkey cells is repaired very poorly after certain kinds of damage. Most recently, it has been found that an actively transcribing gene in $\mathrm{CHO}$ cells is repaired at a much higher rate than other genes. Understanding these novel fime points of DNA repair and how cells p-rioritize the repair of "housekeeping genes" is vital to the interpretation of DNA damage in risk assessment. R. M. Santella and $\mathbf{R}$. Perera discussed the use of monoclonal antibodies for quantitation of damage to both DNA and blood cells, and implications of these measurements to risk assess- 
ment. R. L. Saul and B. N. Ames presented data on the background levels of DNA damage in the population obtained by measuring thymine glycol in urine. R. H. Reitz discussed the role of pharmacokinetics in risk assessment, and $R$. Parshad talked about $G_{2}$ chromosal radiosensitivity, which he associated with deficient DNA repair and cancer susceptibility.

Session X, Risk Assessment and Regulatory Decisions, was chaired by A. D. Upton. Comments on mechanistic considerations were given by Upton. Use of models in low-dose extrapolation (J. Van Ryzin), problems in interspecies extrapolation (R. Wilson), and the time-to-tumor approach in risk assessment and risk management (R. E. Albert) indicated that mechanisms of DNA damage and repair have not become an integral part in current risk assessment considerations. It was also clear that novel scientific approaches in assessing thresholds and risk are badly needed.

Session XI, the last session, dealt with risk assessment of foods, an overview on which was given by $\mathrm{W}$. G. Flamm, session chairman. The role of risk assessment in food safety policy was discussed by R. J. Scheuplein; mutagens and carcinogens in food, by $\mathbf{M}$. J. Prival; and practical aspects of risk assessment of foods, by $\mathbf{R}$. J. Lorentzen. An important role of foods relative to DNA damage was presented by Carmia Borek. She discussed the significance of nutritional factors in cellular protection against DNA damage.

The program of the conference was principally oriented towards relationships between the mechanisms of
However, there are many other diseases, and aging in par-

ticular, in which approaches similar to those reviewed for

cancer should be pursued.

DNA damage and cancer, with cancer prevention in mind. However, there are many other diseases, and aging in particular, in which approaches similar to those reviewed for cancer should be pursued.

The motto of the conference was "DNA is the beginning and the end." Any effort that can postpone

DNA is the beginning and the end.

"the end" and improve the quality of life in the interim by preserving or manipulating the integrity of DNA is worth our unselfish commitment and highest priority.

The proceedings of the conference will be published in a didactic form by Plenum Press in 1986.

Prepared by Michael G. Simic of the Ionizing Radiation Division, National Bureau of Standards, Gaithersburg, MD 20899. 